\title{
THE EULER NUMBER FROM THE DISTANCE FUNCTION
}

\author{
Ximo GUAL-ARnAU \\ Institute of New Imaging Technologies, Campus Riu Sec, Universitat Jaume I, 12071 Castelló, Spain \\ e-mail: gual@uji.es \\ (Received April 11, 2013; revised November 11, 2013; accepted November 12, 2013)
}

\begin{abstract}
We present a new method to obtain the Euler number of a domain based on the tangent counts of concentric spheres in $\mathbb{R}^{3}$ (or circles in $\mathbb{R}^{2}$ ), with respect to the center $O$, that sweeps the domain. This method is derived from the Poincaré-Hopf Theorem, when the index of critical points of the square of the distance function with respect to the origin $O$ is considered.
\end{abstract}

Keywords: critical points, distance function, Euler number, stereology, tangent counts.

\section{INTRODUCTION}

The Euler number describes the topology of the surfaces of a structure of interest and its practical value of obtaining, for example, the number of trabeculae in bone, the number of glomerular capillaries in kidney, or the alveolar number in lung-architecture, has been demonstrated.

Hadwiger (1957) gave an inductive definition of the Euler characteristic which has suggested several methods for deriving the Euler number from close parallel flat sections; see for instance De Hoff (1987), Gundersen et al. (1993), Ohser et al. (1996) or Rataj (2004). In this case, the principle used to obtain the Euler number of an $n$-dimensional domain in $\mathbb{R}^{n}(n=$ 2 or 3$)$ is based on what happens in an $(n-1)$ dimensional plane that sweeps through the domain. Some of the methods obtained from this principle and derived from the Hadwiger definition, can also be proved from the classical Poincaré-Hopf Theorem, when the index of critical points of a family of height functions are considered (Gual-Arnau et al., 2001).

It follows that, for any vector $u$ in $\mathbb{R}^{n}(n=2$ or 3$)$, the Euler number of a domain is obtained from the tangent counts of the domain and a perpendicular plane to $u$ (or line in $\mathbb{R}^{2}$ ), that sweeps the domain. This method is isotropic in the sense that, although the tangent events depend on the direction $u$, the final result (Euler number) does not depend on $u$.

In this paper we present an alternative method to obtain the Euler number of a domain based on the tangent counts of concentric spheres in $\mathbb{R}^{3}$ (or circles in $\mathbb{R}^{2}$ ) that sweep the domain. The tangent counts will depend on the position of the center $O$ of the spheres (or circles) but the final result will be the Euler number for a general position of the center $O$. This method is then based on what happens in a sphere in $\mathbb{R}^{3}$ (or circle in $\mathbb{R}^{2}$ ) that sweeps through the domain and it is obtained from the Poincaré-Hopf Theorem, when the index of critical points of the square of the distance function with respect to an origin $O$ is considered. In fact, Chapter 6 of Milnor (1969) is deserved to show that the square distance from a chosen point, restricted to a smooth submanifold in $\mathbb{R}^{n}$, is indeed a function with no degenerate critical points (Morse function), for almost all chosen points for the origin $O$. For instance, if the domain is bounded by a sphere, the square distance restricted to the sphere is a Morse function for all reference points, except when the reference point $O$ is the center of the sphere.

This method may be of interest in images with a prominent reference point: e.g., the nucleolus of a cell or the planet earth when counting celestial objects like stars; and in images which include anisotropic particles that have approximately parallel sides (plant fibers, fibrous minerals,...).

In the first part of the paper we give the stereological versions of the principle for 3dimensional domains in $\mathbb{R}^{3}$, 2-dimensional domains in $\mathbb{R}^{2}$ and domains with boundary in a surface $S \subset \mathbb{R}^{3}$. In the second part we give the detailed mathematical justifications and we relate the EulerPoincare characteristic with curvatures of the domain and the concentric spheres in $\mathbb{R}^{3}$ (or circles in $\mathbb{R}^{2}$ ).

\section{STEREOLOGICAL FORMULAE}

In this section we give an elementary version of the Euler-Poincaré characteristic of closed orientable smooth surfaces $S=\partial D$ in $\mathbb{R}^{3}$, of bounded domains $D \in \mathbb{R}^{2}$, and of domains with boundary in an orientable smooth surface $S$ in $\mathbb{R}^{3}$. 
The Euler-Poincaré characteristic for domains in $\mathbb{R}^{2}$ and $\mathbb{R}^{3}$ has been studied in several stereological applications (see, for instance, Gundersen et al., 1993). The principle used to obtain the Euler number of an $n$-dimensional domain in $\mathbb{R}^{n}(n=2$ or 3$)$, is based on what happens in an $(n-1)$-dimensional plane that sweeps through the domain. Here we consider a new principle based on what happens in a sphere $\mathbb{S}^{2}(\lambda)$ (or circle $\mathbb{S}^{1}(\lambda)$ in $\mathbb{R}^{2}$ ) that sweeps through the domain when the radius $\lambda$ varies.

Both principles can be derived from a result of Morse (1929) that we simplify for surfaces in $\mathbb{R}^{3}$ and domains in $\mathbb{R}^{2}$. In the next section we give a more detailed mathematical justification.

\section{CLOSED SURFACES IN $\mathbb{R}^{3}$}

Let $f: S \longrightarrow \mathbb{R}$ be a smooth function defined on a closed orientable smooth surface. We say that $x \in S$ is a critical point if $\nabla f_{x}: T_{x} S \longrightarrow \mathbb{R}$ vanishes for every $v \in T_{x} S$. A critical point $x \in S$ is non degenerated if the Hessian of $f$ at $x,\left(\nabla^{2} f\right)_{x}: T_{x} S \times T_{x} S \longrightarrow \mathbb{R}$, is a non degenerated bilinear form, i.e., for every non zero vector $v \in T_{x} S$, there exists a vector $w \in T_{x} S$ such that $\left(\nabla^{2} f\right)_{x}(v, w) \neq 0$. A function $\mathrm{f}$ is called a Morse function if all its critical points are non-degenerated (Bruce et al., 1984).

Theorem 1. (Morse) Let $f: S \rightarrow \mathbb{R}$ be a differentiable Morse function on a closed orientable two-dimensional surface, then

$$
\chi(S)=\sum_{x \in \Sigma(f)} \operatorname{Ind}_{x}(f),
$$

where $\Sigma(f)$ denotes the set of singular points of $f$ and the index, $\operatorname{Ind}_{x}(f)$, is given by +1 when $x$ is a local extreme of $f$, or -1 when $x$ is a saddle point (Morse, 1929).

That is,

$$
\chi(S)=M-s+m,
$$

where $M, m$ and $s$ denote the number of maximum, minimum and saddle, respectively, of $f$.

Now, we apply the above results to a particular function: the square of the distance function.

We define the square of the distance function to a generic coordinate origin $O \in \mathbb{R}^{3}$ as

$$
\begin{aligned}
d & : \mathbb{R}^{3} \longrightarrow \mathbb{R} \\
& x \longrightarrow d(x)=\langle x, x\rangle=\|x\|^{2} .
\end{aligned}
$$

For almost all $O \in \mathbb{R}^{3}$ (all but a set of measure 0 ), the restriction of $d$ to $S,\left.d\right|_{S}$, is a Morse function
(Milnor, 1969). Now we will apply Theorem 1 to the square of the distance function.

The level sets are $\pi_{\lambda^{2}}=d^{-1}\left(\lambda^{2}\right)=\{x \in$ $\left.\mathbb{R}^{3} /\|x\|^{2}=\lambda^{2}\right\}=\mathbb{S}^{2}(\lambda)$.

When we restrict $d$ to $S$, the critical points $x \in$ $\Sigma\left(\left.d\right|_{S}\right)$ are those where the tangent plane to $S$ at $x$ coincides with the tangent plane to $\pi_{\lambda^{2}}$ at $x$; i.e., $T_{x} S=$ $T_{x} \mathbb{S}^{2}(\lambda)$; then we give a 'stereological' interpretation of the local extreme (maximum and minimum) and saddle points of $d$.

Let $D$ be a domain in $\mathbb{R}^{3}$ such that $\partial D=S$; then, the critical points of $\left.d\right|_{S},\left(x \in \Sigma\left(\left.d\right|_{S}\right)\right)$, can be classified as follows: $\left.x \in \Sigma\left(\left.d\right|_{S}\right)\right)$ is a point of type "Island" if $d^{-1}(d(x))=\mathbb{S}^{2}(\lambda)$ is locally contained in $\mathbb{R}^{3} \backslash D,(x$ is a local extreme (maximum) of $\left.\left.d\right|_{S}\right) ; x \in \Sigma\left(\left.d\right|_{S}\right)$ ) is a point of type "Hole" if $d^{-1}(d(x))$ is locally contained in $D$ ( $x$ is a local extreme (minimum) of $\left.d\right|_{S}$ ); and $\left.x \in \Sigma\left(\left.d\right|_{S}\right)\right)$ is a point of type "Bridge" if there exist points of $S$ in the interior and in the exterior of $\mathbb{S}^{2}(\lambda)$ where $\mathbb{S}^{2}(\lambda)=d^{-1}(d(x)),\left(x\right.$ is a saddle point of $\left.\left.d\right|_{S}\right)$, (see Fig. 1).
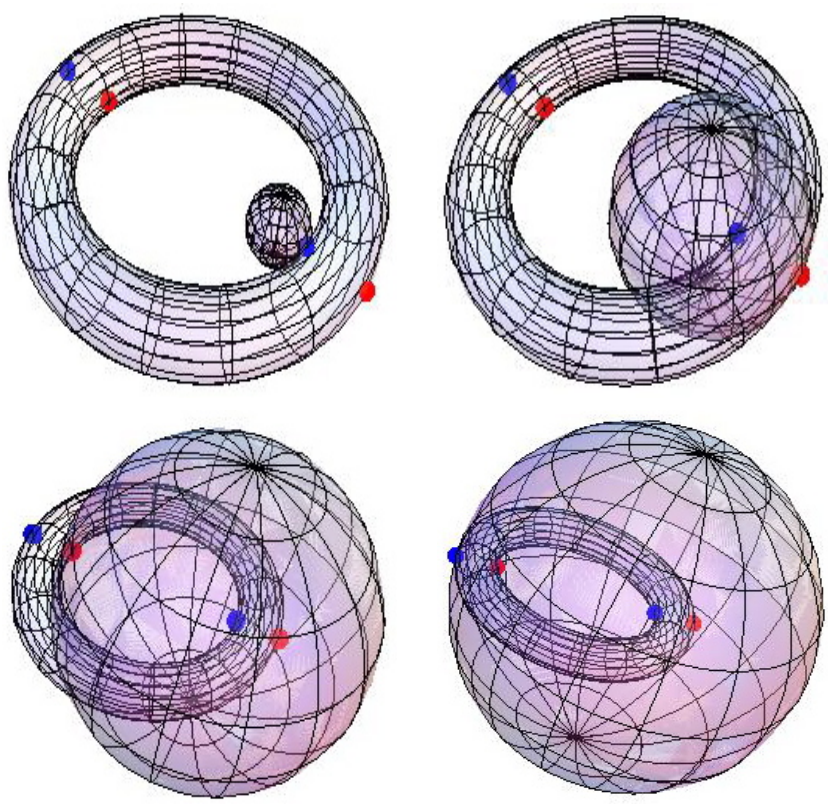

Fig. 1. Images that we see in the sweeping spheres $\mathbb{S}^{2}(\lambda) \cap S$, where $S$ is a fixed torus and the growing spheres $\mathbb{S}^{2}(\lambda)$ have a fixed center $O$. Depending on the type of critical point we have Islands or Holes (blue points) and Bridges (red points). The Euler number of the torus is 0 . Note that, although $S$ is fixed, as the radius of $\mathbb{S}^{2}(\lambda)$ increases, a change of scale and orientation of $S$ (torus) appears in the images to get a better view of the critical (tangent) point. 
Let $S=\partial D \subset \mathbb{R}^{3}$ be an orientable smooth surface in $\mathbb{R}^{3}$ and let $O \in \mathbb{R}^{3}$ such that $\left.d\right|_{S}$ is a Morse function. When $\lambda$ varies on $\mathbb{R}$, the different spheres $\pi_{\lambda^{2}}$ can be considered as a "sweeping" sphere $\mathbb{S}^{2}(\lambda)$ (centered at $O$ ) in $\mathbb{R}^{3}$. Then, Theorem 1, with the interpretation of local extrema and saddle points of $\left.d\right|_{S}$ as Islands, Holes and Bridges, can be expressed as:

$$
\chi(S)=2 \chi(D)=I_{2}+H_{2}-B_{2},
$$

where $I_{2}, H_{2}, B_{2}$ denote the number of islands, holes and bridges observed in the "sweeping" sphere $\mathbb{S}^{2}(\lambda)$, which contribute to the sum $\sum_{x \in \Sigma\left(\left.d\right|_{S}\right)} \operatorname{Ind}_{x}\left(\left.d\right|_{S}\right)$.

Moreover, when $O \in S, O$ is a minimum of $d$, and

$$
\chi(S)=2 \chi(D)=1+I_{2}+H_{2}-B_{2} .
$$

Note that the tangent counts $I_{2}, H_{2}, B_{2}$ may depend on the position of the center $O$; however the final result of $\left(I_{2}+H_{2}-B_{2}\right)$ is always $2 \chi(D)$ (see Fig. 2). In fact, when $O$ is far from the domain $D$ the tangent counts $I_{2}, H_{2}, B_{2}$ are similar to that obtained when sweeping planes are considered.
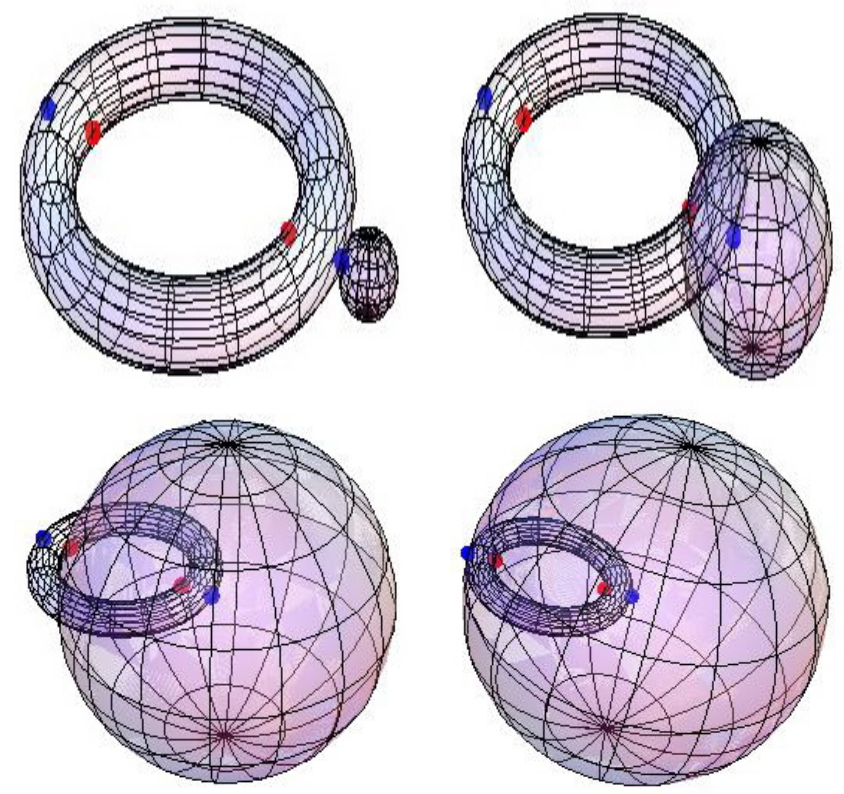

Fig. 2. The surface $S$ is the same as in Fig. 1 and the growing spheres $\mathbb{S}^{2}(\lambda)$ have a fixed origin O different to that considered in Fig. 1. Therefore, we obtain different values of the critical points, because they depend on the position of the center of the spheres, $O$. Blue points are Islands and red points are Bridges. The Euler number of the torus is 0 .

\section{DOMAINS IN $\mathbb{R}^{2}$}

Let $D \subset \mathbb{R}^{2}$ be a domain in $\mathbb{R}^{2}$ whose boundary is a curve $\partial D$. In this case, we can not apply Theorem 1 because $D$ is a surface with a boundary. As explained in the Mathematical foundations section, the generalized result of Theorem 1 for domains in $\mathbb{R}^{2}$ is as follows:

Theorem 2. Let $D \subset \mathbb{R}^{2}$ be a domain with boundary and let $f: D \rightarrow \mathbb{R}$ be a Morse function such that $f$ has no critical points on $\partial D$ and the restriction $\left.f\right|_{\partial D}: \partial D \rightarrow \mathbb{R}$ is also a Morse function. Then,

$$
\chi(D)=\sum_{x \in \Sigma(f)} \operatorname{Ind}_{x}(f)+\frac{1}{2} \sum_{y \in \Sigma\left(\left.f\right|_{\partial D}\right)} \operatorname{Ind}_{y}(f),
$$

where the index $\operatorname{Ind}_{x}(f)$ is defined as in Eq. 1 and the index $\operatorname{Ind}_{x}\left(\left.f\right|_{\partial D}\right)$ is defined as follows: if the level set $f^{-1}(f(y))$ is locally contained in $\mathbb{R}^{2} \backslash D$ then $\operatorname{Ind}_{y}\left(\left.f\right|_{\partial D}\right)=+1$ and if the level set $f^{-1}(f(y))$ is locally contained in $D$ then $\operatorname{Ind}_{y}\left(\left.f\right|_{\partial D}\right)=-1$.

The square of the distance function to a generic coordinate origin $O \in \mathbb{R}^{2}$ is

$$
\begin{aligned}
& d: \mathbb{R}^{2} \longrightarrow \mathbb{R} \\
& \quad x \longrightarrow d(x)=\langle x, x\rangle=\|x\|^{2} .
\end{aligned}
$$

The level curves $\pi_{\lambda^{2}}$ are in this case circles $\mathbb{S}^{1}(\lambda)$ centered at $O \in \mathbb{R}^{2}$.

To satisfy Theorem 2 we suppose that $O \in \mathbb{R}^{2} \backslash$ $\partial D$; then, the square of the distance function $\left.d\right|_{D}$ has only a critical point $O \in D$; and we also suppose that the restriction of the square of the distance function $\left.d\right|_{\partial D}$ is a Morse function; then, from Eq. 6,

$$
\chi(D)=\delta_{0}(D)+\frac{1}{2} \sum_{y \in \Sigma\left(\left.d\right|_{\partial D}\right)} \operatorname{Ind}_{y}\left(\left.d\right|_{\partial D}\right),
$$

where

$$
\delta_{0}(D)=\left\{\begin{array}{l}
1, \text { if } O \in D, \\
0, \text { if } O \notin D,
\end{array}\right.
$$

and a critical point $y \in \Sigma\left(\left.d\right|_{\partial D}\right)$ is a point of type "Island" if $d^{-1}(d(y))=\mathbb{S}^{1}(\lambda)$ is locally contained in $\mathbb{R}^{2} \backslash D$; and $\left.y \in \Sigma\left(\left.d\right|_{\partial D}\right)\right)$ is a point of type "Bridge" if $d^{-1}(d(y))$ is locally contained in $D$ (see Fig. 3 ).

Therefore, when $\lambda$ varies on $\mathbb{R}$, the different circles $\mathbb{S}^{1}(\lambda)$ can be considered as "sweeping" circles in $\mathbb{R}^{2}$, and Eq. 6 can be expressed as:

$$
\chi(D)=\delta_{0}(D)+\frac{1}{2}\left(I_{1}-B_{1}\right),
$$

where $I_{1}$ and $B_{1}$ denote the number of islands and bridges observed in the "sweeping" circle $\mathbb{S}^{1}(\lambda)$. 


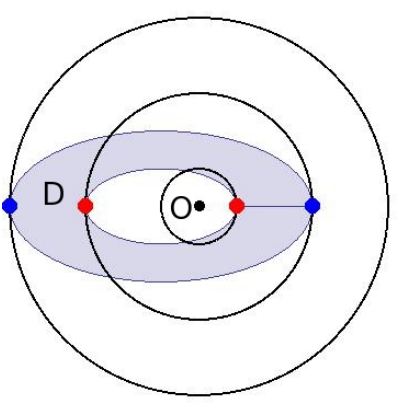

a) $O \notin D$.

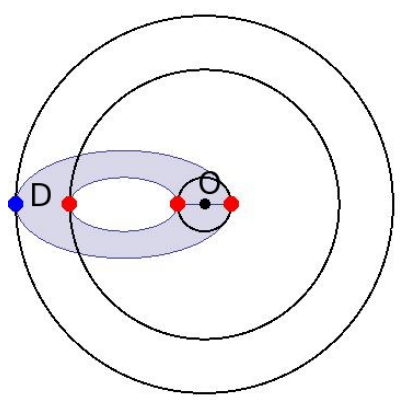

b) $O \in D$.
Fig. 3. Different values of the critical points, depending on the position of the center of the growing circles, $O$. Blue points are Islands and red points are Bridges. The Euler number of the domain $D$ is 0 .

Note that when the point $O \notin D$, Eq. 10 can be expressed as:

$$
\chi(D)=\frac{1}{2}\left(I_{1}-B_{1}\right)
$$

which, when $O$ is far from $D$, is similar to that obtained in Gundersen et al. (1993), where $I_{1}$ and $B_{1}$ denote the number of islands and bridges observed in a "sweeping" line.

\section{SURFACES WITH BOUNDARY IN $\mathbb{R}^{3}$}

Let $D \subset S \subset \mathbb{R}^{3}$ be a domain with boundary in an orientable smooth surface $S$ in $\mathbb{R}^{3}$ and let $O \in \mathbb{R}^{3}$ such that Eq. 14 is satisfied in this case. When $\lambda$ varies on $\mathbb{R}^{+}$, the different spheres $\mathbb{S}^{2}(\lambda)$ can be considered as a sweeping sphere in $\mathbb{R}^{3}$, and

$$
\chi(D)=\left(I_{2}-B_{2}\right)+\frac{1}{2}\left(I_{1}-B_{1}\right),
$$

where $I_{2}, B_{2}$ are defined as in Eq. 4 and $I_{1}, B_{1}$ denote the number of islands and bridges, respectively, observed in the level curves $\mathbb{S}^{2}(\lambda) \cap S$. In Fig. 4 we consider a domain $D$ in a cylinder, whose boundary is given by two circles (black curves), and we show two critical points: an Island where the level curve $S \cap \mathbb{S}^{2}(\lambda)$ (red curve) is locally contained in $S \backslash D$, and a Bridge point, where the level curve is locally contained in $D$.

\section{MATHEMATICAL FOUNDATIONS}

The classical Poincaré-Hopf Theorem states that if $S$ is a closed orientable smooth surface and $v$ is a smooth vector field on $S$ with isolated zeros, then

$$
\chi(S)=\sum_{v(x)=0} \operatorname{Ind}_{x}(v)
$$

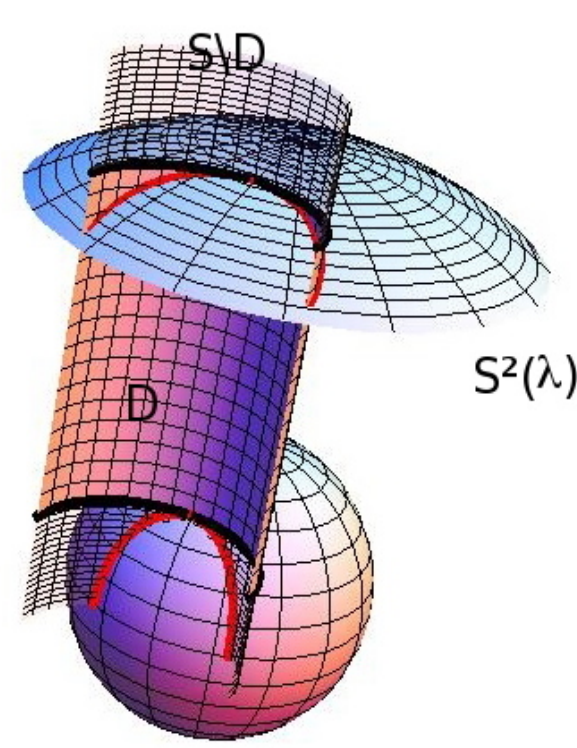

Fig. 4. Partial view of the domain $D$. The boundary $\partial D$ is given by the black curves (circles). The red curves correspond to $S \cap \mathbb{S}^{2}(\lambda)$.

where $\chi(S)$ is the Euler-Poincaré characteristic of $S$ and the index, $\operatorname{Ind}_{x}(v)$, is just the local degree of $v$ at $x$; that is, the degree of the map $u: S \longrightarrow \mathbb{S}^{2}$ given by $u(z)=v(z) /|v(z)|$. As an immediate consequence, we see that if $f: S \rightarrow \mathbb{R}$ is a Morse function (that is, a function with non-degenerate critical points), then

$$
\chi(S)=\sum_{x \in \Sigma(f)} \operatorname{Ind}_{x}(f)
$$

where $\Sigma(f)$ denotes the set of singular points of $f$ and the index, $\operatorname{Ind}_{x}(f)$, is given by +1 when $x$ is a local extreme of $f$, or -1 when $x$ is a saddle point (Morse, 1929).

Now, we consider a generalization of the classical Poincaré-Hopf Theorem which can be applied domains with boundary in an orientable smooth surface in $\mathbb{R}^{3}$ and, in particular, to closed domains in $\mathbb{R}^{2}$ (Gual-Arnau et al., 2001).

Let $D \subset \mathbb{R}^{3}$ be a compact orientable smooth surface with boundary and let $f: D \rightarrow \mathbb{R}$ be a Morse function such that $f$ has no critical points on $\partial D$ and the restriction $\left.f\right|_{\partial D}: \partial D \rightarrow \mathbb{R}$ is also a Morse function. Then, the Poincaré-Hopf Theorem (Morse, 1929), states

$$
\chi(D)=\sum_{x \in \Sigma(f)} \operatorname{Ind}_{x}(f)+\frac{1}{2} \sum_{y \in \Sigma\left(\left.f\right|_{\partial D}\right)} \operatorname{Ind}_{y}(f)
$$

where the index $\operatorname{Ind}_{x}(f)$ is defined as in Eq. 1 and the index $\operatorname{Ind}_{x}\left(\left.f\right|_{\partial D}\right)$ is defined as follows: if the level set $f^{-1}(f(y))$ is not locally contained in $D$ then 
$\operatorname{Ind}_{y}\left(\left.f\right|_{\partial D}\right)=+1$ and if the level set $f^{-1}(f(y))$ is locally contained in $D$ then $\operatorname{Ind}_{y}\left(\left.f\right|_{\partial D}\right)=-1$.

In the next section we will consider a particular function $f$, the square of the distance function, and we will interpret Eq. 1 in terms of critical points of this function.

\section{CLOSED SURFACES IN $\mathbb{R}^{3}$}

Let $S$ be a closed orientable smooth surface in $\mathbb{R}^{3}$. Given $x \in S$, we denote by $N(x)$ and $K(x)$ the normal vector and the Gauss curvature of $S$, respectively.

Theorem 3. Let $S \subset \mathbb{R}^{3}$ be a closed orientable smooth surface in $\mathbb{R}^{3}$.

1. Let $x \in S$. It is a critical point of $\left.d\right|_{S}$ if and only if $x= \pm \lambda N(x)$ if and only if $T_{x} S=T_{x} \mathbb{S}^{2}(\lambda)$ (where $\left.d(x)=\lambda^{2}\right)$.

2. Let $x \in S$ be a critical point of $\left.d\right|_{S}$.

(a) Suppose that $x=-\lambda N(x)$; then $x$ is nondegenerate if and only if $\kappa_{1} \neq \frac{1}{\lambda}$ and $\kappa_{2} \neq \frac{1}{\lambda}$ $\left(\frac{1}{\lambda^{2}}\right.$ is the Gauss curvature of $\mathbb{S}^{2}(\lambda)$ ). Moreover, it is a local extreme or a saddle point of $\left.d\right|_{S}$ depending on weather $\left(\frac{1}{\lambda}-\kappa_{1}\right)\left(\frac{1}{\lambda}-\kappa_{2}\right)$ is positive or negative, respectively.

(b) Suppose that $x=\lambda N(x)$; then $x$ is nondegenerate if and only if $\kappa_{1} \neq-\frac{1}{\lambda}$ and $\kappa_{2} \neq$ $-\frac{1}{\lambda}$. Moreover, it is a local extreme or a saddle point of $\left.d\right|_{S}$ depending on weather $\left(\frac{1}{\lambda}+\kappa_{1}\right)\left(\frac{1}{\lambda}+\kappa_{2}\right)$ is positive or negative, respectively.

Proof. To prove part 1 we consider

$$
\begin{aligned}
(\nabla d)_{x}(v) & =\left.\frac{d}{d t}\right|_{t=0}\|\alpha(t)\|^{2} \\
& =2\left\langle\alpha^{\prime}(0), \alpha(0)\right\rangle=2\langle v, x\rangle,
\end{aligned}
$$

where $\alpha:(-\varepsilon, \varepsilon) \longrightarrow S$ is a differentiable curve with $\alpha(0)=x$ and $\alpha^{\prime}(0)=v$. Then, $(\nabla d)_{x}(v)=0$ if and only if $v \perp x$ if and only if $x= \pm \lambda N(x)$ if and only if $N(x) \perp T_{x} \mathbb{S}^{2}(\lambda)(\|x\|=\lambda)$ if and only if $T_{x} S=$ $T_{x} \mathbb{S}^{2}(\lambda)$. An alternative proof using local coordinates can be found in Milnor (1969).

To prove part 2a we consider the Hessian of $d$ at $x$,

$$
\begin{aligned}
\left(\nabla^{2} d\right)_{x}(v) & =\left.\frac{d^{2}}{d t^{2}}\right|_{t=0}|| \alpha(t) \|^{2}=\left.2 \frac{d}{d t}\right|_{t=0}\left\langle\alpha^{\prime}(t), \alpha(t)\right\rangle \\
& =2\left(\left\langle\alpha^{\prime \prime}(0), \alpha(0)\right\rangle+\left\langle\alpha^{\prime}(0), \alpha^{\prime}(0)\right\rangle\right) \\
& =2\left(\left\langle\alpha^{\prime \prime}(0),-\lambda N(x)\right\rangle+\langle v, v\rangle\right) .
\end{aligned}
$$

Now, if we take derivatives at $t=0$ of the identity $\left\langle\alpha^{\prime}(t),-\lambda N(\alpha(t))\right\rangle=0$ we obtain

$$
\left\langle\alpha^{\prime \prime}(0),-\lambda N(x)\right\rangle=-\langle v,-\lambda d N(x)(v)\rangle .
$$

Since the second fundamental form of $S$ at $x$ is defined as $I I_{x}(v, v)=-\langle d N(x)(v), v\rangle$ and it is symmetric, we obtain

$$
\left(\nabla^{2} d\right)_{x}(v)=2\left(\|v\|^{2}-\lambda I I_{x}(v, v)\right) .
$$

Let $\left\{e_{1}, e_{2}\right\}$ be a principal orthonormal frame at $x$; then, in matrix form we have

$$
\left(\nabla^{2} d\right)_{x}(v)=v\left(\begin{array}{cc}
1-\lambda \kappa_{1} & 0 \\
0 & 1-\lambda \kappa_{2}
\end{array}\right) v^{T} .
$$

So, $x$ is non-degenerate if $\left(1-\lambda \kappa_{1}\right)\left(1-\lambda \kappa_{2}\right) \neq 0$; that is, $\kappa_{1} \neq \frac{1}{\lambda}$ and $\kappa_{2} \neq \frac{1}{\lambda}$. The rest of part $2 \mathrm{a}$ is derived from the classification of local extrema and the proof of part $2 \mathrm{~b}$ is similar.

Corollary 1. Let $S \subset \mathbb{R}^{3}$ be a closed orientable smooth surface in $\mathbb{R}^{3}$. Then

$$
\begin{aligned}
\chi(S) & =\sum_{x \in S / x=-\lambda N(x)} \operatorname{sign}\left(1-\lambda \kappa_{1}\right)\left(1-\lambda \kappa_{2}\right) \\
& +\sum_{x \in S / x=\lambda N(x)} \operatorname{sign}\left(1+\lambda \kappa_{1}\right)\left(1+\lambda \kappa_{2}\right) \\
= & \sum_{x \in S / x=-\lambda N(x)} \operatorname{sign}\left(1-2 \lambda H(x)+\lambda^{2} K(x)\right) \\
& +\sum_{x \in S / x=\lambda N(x)} \operatorname{sign}\left(1+2 \lambda H(x)+\lambda^{2} K(x)\right) .
\end{aligned}
$$

where $H(x)$ denotes the mean curvature of $S$ at $x$.

Proof. This formula is a direct consequence of Eq. 1 and Theorem 3.

Remark. In Milnor (1969) it is proved that for almost all $O \in \mathbb{R}^{3}$ the function $\left.d\right|_{S}$ is a Morse function; i.e., in general, all the critical points of $\left.d\right|_{S}$ are nondegenerate (these points are characterized in Theorem 3). When $O \in S$, from Eqs. 15 and 16, we have that $\left.d\right|_{S}$ has a non-degenerate critical point (minimum) at $x=O$. In this case $\operatorname{Ind}_{O}\left(\left.d\right|_{S}\right)=+1$.

\section{DOMAINS IN $\mathbb{R}^{2}$}

Let $D \subset \mathbb{R}^{2}$ be a domain with boundary. As in the preceding section, we consider now the square of the distance function $d: \mathbb{R}^{2} \rightarrow \mathbb{R}$, instead of the square of the distance function of $\mathbb{R}^{3}$. The level curves $\pi_{\lambda^{2}}$ are in this case circles $\mathbb{S}^{1}(\lambda)$.

We suppose that the origin $O \in \mathbb{R}^{2} \backslash \partial D$, then, the square of the distance function $\left.d\right|_{D}$ only has the point $O$ as critical point (minimum) if $O \in D$ (the distance function $d$ defined in $D$ is given now by $d(x, y)=$ $x^{2}+y^{2}$; and this function has a minimum at the point 
$O=(0,0) \in D)$. Moreover, in general, the restriction of the square of the distance function $\left.d\right|_{\partial D}$ is a Morse function; then, from Eq. 14,

$$
\chi(D)=\delta_{0}(D)+\frac{1}{2} \sum_{y \in \Sigma\left(\left.d\right|_{\partial D}\right)} \operatorname{Ind}_{y}\left(\left.d\right|_{\partial D}\right),
$$

where

$$
\delta_{0}(D)=\left\{\begin{array}{l}
1, \text { if } O \in D, \\
0, \text { if } O \notin D .
\end{array}\right.
$$

Lemma 1. Let $D \subset \mathbb{R}^{2}$ be a domain with boundary; then,

$$
\begin{aligned}
\chi(D)=\delta_{0}(D) & +\frac{1}{2}\left(\sum_{y \in \partial D / y=-\lambda n(y)} \operatorname{sign}\left(\kappa-\frac{1}{\lambda}\right)\right. \\
& \left.+\sum_{y \in \partial D / y=\lambda n(y)} \operatorname{sign}\left(\kappa+\frac{1}{\lambda}\right)\right),
\end{aligned}
$$

where $n(y)$ and $\kappa(y)$ are the normal vector and the curvature of the plane curve $\partial D$, respectively.

Proof. Suppose that $\alpha$ is a parameterization by arc length of $\partial D$. Given a point $y \in \Sigma\left(\left.d\right|_{\partial D}\right)$, with $\alpha\left(s_{0}\right)=y$, we have $\operatorname{Dd}\left(s_{0}\right)=\left\langle\alpha^{\prime}\left(s_{0}\right), \alpha\left(s_{0}\right)\right\rangle=0$, then $\alpha^{\prime}\left(s_{0}\right)$ is tangent to $S^{1}(\lambda)$ at $y$. Moreover, $D^{2} h_{u}\left(s_{0}\right)=\left\langle\alpha^{\prime \prime}\left(s_{0}\right), \alpha\left(s_{0}\right)\right\rangle+\left\langle\alpha^{\prime}\left(s_{0}\right), \alpha^{\prime}\left(s_{0}\right)\right\rangle=$ $\langle\kappa(y) n(y), \pm \lambda n(y)\rangle+1=1 \pm \lambda \kappa(y)$ so $y$ is a local extreme. Finally, from the way we have chosen the orientation on $\partial D$ and the definition of $\operatorname{Ind}_{y}\left(\left.h_{u}\right|_{\partial D}\right)$ given in Eq. 14, we have the result.

\section{SURFACES WITH BOUNDARY IN $\mathbb{R}^{3}$}

Let $D \subset S \subset \mathbb{R}^{3}$ be a domain with boundary in an orientable smooth surface $S$ in $\mathbb{R}^{3}$ (Gual-Arnau et al., 2001). Parts 1 and 2 of Theorem 3 are valid here when we restrict $\left.d\right|_{D}$ and $x \in D$.

We will give a geometrical interpretation of the critical points of $\left.d\right|_{\partial D}$. The level sets $S \cap \mathbb{S}^{2}(\lambda)$ and $D \cap \mathbb{S}^{2}(\lambda)$ are now curves in $S$. Given $y \in \partial D$, let $n(y)$ and $\kappa_{g}(y)$ denote the normal vector and the geodesic curvature of $\partial D$ in $D$ at $y$, respectively. We consider the orientation in $\partial D$ such that $n(y)$ points to the interior of $D$. Let $\kappa_{g}^{0}(y)$ denote the geodesic curvature of the level curve $D \cap \mathbb{S}^{2}(\lambda)$ at a regular point $y$ of $\left.d\right|_{D}$. If the curves $S \cap \mathbb{S}^{2}(\lambda)$ and $\partial D$ are tangent at $y$ we choose in $S \cap \mathbb{S}^{2}(\lambda)$ the same orientation.

Theorem 4. Let $D$ be a domain with boundary in an orientable smooth surface $S$ in $\mathbb{R}^{3}$.

1. Let $y \in \partial D$ be a regular point of $\left.d\right|_{D}$. $y$ is a critical point of $\left.d\right|_{\partial D}$ if and only if the curves $D \cap \mathbb{S}^{2}(\lambda)$ and $\partial D$ are tangent at $y$.
2. Let $y \in \partial D$ be a regular point of $\left.d\right|_{D}$ and a critical point of $\left.d\right|_{\partial D} . y$ is non-degenerate if and only if $\kappa_{g}(y) \neq \kappa_{g}^{0}(y)$. Moreover, it is an island when $\left.\kappa_{g}(y)\right\rangle \kappa_{g}^{0}(y)$ and it is a bridge when $\kappa_{g}(y)\left\langle\kappa_{g}^{0}(y)\right.$.

Proof. Suppose that $\beta(s)$ is a parameterization of $S \cap \mathbb{S}^{2}(\lambda)$ by arc length with $\alpha\left(s_{0}\right)=y$. The vector $\mathrm{y}$ can be expressed as

$$
\begin{aligned}
y= & \langle y, N(y)\rangle N(y)+\left\langle y, \beta^{\prime}\left(s_{0}\right)\right\rangle \beta^{\prime}\left(s_{0}\right) \\
& +\left\langle y, N(y) \wedge \beta^{\prime}\left(s_{0}\right)\right\rangle N(y) \wedge \beta^{\prime}\left(s_{0}\right) .
\end{aligned}
$$
0 .

Since $\langle\beta(s), \beta(s)\rangle=\lambda^{2}$ we have that $\left\langle\beta^{\prime}\left(s_{0}\right), y\right\rangle=$

Suppose now that $\alpha(s)$ is a parameterization of $\partial D$ by arc length with $\beta\left(s_{0}\right)=y$. Then,

$$
\begin{aligned}
y= & \langle y, N(y)\rangle N(y)+\left\langle y, \alpha^{\prime}\left(s_{0}\right)\right\rangle \alpha^{\prime}\left(s_{0}\right) \\
& +\left\langle y, N(y) \wedge \alpha^{\prime}\left(s_{0}\right)\right\rangle N(y) \wedge \alpha^{\prime}\left(s_{0}\right),
\end{aligned}
$$

where $N(y) \wedge \alpha^{\prime}\left(s_{0}\right)=n(y)$.

$y$ is a critical point of $\left.d\right|_{\partial D}$ if $\left\langle y, \alpha^{\prime}\left(s_{0}\right)\right\rangle=0$. Since $y$ is a regular point of $\left.d\right|_{D}$, from Theorem 3 we have that $y \neq \lambda N(y)$; then, from Eqs. 23 and 24, $y$ is a critical point of $\left.d\right|_{\partial D}$ if and only if the normal vectors to $\alpha$ and $\beta$ in $T_{y} \partial D$ coincide, and therefore the curves $D \cap \mathbb{S}^{2}(\lambda)$ and $\partial D$ are tangent at $y$.

To prove the second part of the theorem we suppose that $y=\alpha\left(s_{0}\right)$ is a critical point of $\left.d\right|_{\partial D}$, i.e., $\left\langle\alpha^{\prime}\left(s_{0}\right), \alpha\left(s_{0}\right)\right\rangle=0$.

$y$ will be a degenerate critical point of $\left.d\right|_{\partial D}$ if

$$
\left\langle\alpha^{\prime \prime}\left(s_{0}\right), \alpha\left(s_{0}\right)\right\rangle+\left\langle\alpha^{\prime}\left(s_{0}\right), \alpha^{\prime}\left(s_{0}\right)\right\rangle=0,
$$

that is, $\left\langle\alpha^{\prime \prime}\left(s_{0}\right), \alpha\left(s_{0}\right)\right\rangle=-1$.

Since $\langle\beta(s), \beta(s)\rangle=\lambda^{2}$ we have that $\left\langle\beta^{\prime \prime}\left(s_{0}\right), \beta\left(s_{0}\right)\right\rangle=-1$.

On the other hand,

$$
\begin{aligned}
& \left\langle\alpha^{\prime \prime}\left(s_{0}\right), y\right\rangle=\kappa_{n}(y)\langle y, N(y)\rangle+\kappa_{g}(y)\langle y, n(y)\rangle, \\
& \left\langle\beta^{\prime \prime}\left(s_{0}\right), y\right\rangle=\kappa_{n}(y)\langle y, N(y)\rangle+\kappa_{g}^{0}(y)\langle y, n(y)\rangle,
\end{aligned}
$$

where the normal curvature $\kappa_{n}(y)$ of $\alpha$ at $y$ coincides, from the Meusnier's theorem, with the normal curvature of $\beta$ at $y$. Then, subtracting Eq. 26 to Eq. 25, we obtain

$$
\left\langle\alpha^{\prime \prime}\left(s_{0}\right), y\right\rangle+1=\left(\kappa_{g}(y)-\kappa_{g}^{0}(y)\right)\langle y, n(y)\rangle .
$$

Finally, since $\langle y, n(y)\rangle \neq 0$, we conclude that $y$ is non-degenerate if and only if $\kappa_{g}(y) \neq \kappa_{g}^{0}(y)$. The fact that the cases $\left.\kappa_{g}(y)\right\rangle \kappa_{g}^{0}(y)$ and $\kappa_{g}(y)\left\langle\kappa_{g}^{0}(y)\right.$ correspond to an island or bridge, respectively, can be deduced from the orientation we have chosen on $\partial D$. 


\section{ACKNOWLEDGEMENTS}

This research was supported by the Spanish Ministry of Education and Science I+D Project MTM2009-14500-C02-02, the UJI project P11B201224 and the PROMETEO/2010/028 project.

\section{REFERENCES}

Bruce JW, Giblin PJ (1984). Curves and singularities. Cambridge: Cambridge University Press.

De Hoff RT (1987). Use of the disector to estimate the Euler characteristics of three-dimensional micro structures. Acta Stereol 6:133-40.

Gual-Arnau X, Nuño-Ballesteros JJ (2001). A stereological version of the Gauss-Bonnet formula. Geometriae Dedicata 84:253-60.

Gundersen HJG, Boyce RW, Nyengaard JR, Odgaard A (1993). The conneulor: unbiased estimation of connectivity using physical disectors under projection. Bone 14:217-22.

Hadwiger H (1957). Vorlesungen über Inhalt, Oberfläche und Isoperimetrie. Berlin: Springer Verlag.

Milnor JW (1969). Morse theory. Princeton University Press.

Morse M (1929). Singular points of vector fields under general boundary conditions. Amer J Math 51:165-78.

Ohser J, Nagel W (1996). The estimation of the EulerPoincaré characteristic from observations on parallel sections. J Microsc 184:117-26.

Rataj J (2004). On estimation of the Euler number by projections on thin slabs. Adv Appl Prob (SGSA) 36:715-24.

Santaló LA (1976). Integral geometry and geometric probability. London: Addison-Wesley.

Teufel E (1982). Differential topology and the computation of total absolute curvature. Math Ann 258:471-80. 